\title{
Automated Iris Recognition System: An Overview
}

\author{
Bhagyashri S. Satpute \\ ME student \\ JSPM's RSCOE, Tathawde, Pune \\ Department of Electronics and Telecommunication
}

\author{
B. D. Jadhav \\ Assistant Professor \\ Department of Electronics and Telecommunication \\ JSPM's RSCOE, Tathawde, Pune
}

\begin{abstract}
Iris texture pattern can be used for biometric verification and identification of a person from a large dataset. Iris recognition is used in several fields, like border, security prone, industrial and medical institutes etc. Due to its high correctness and uniqueness, it is used in several fields of access control and border area security. The demand for iris recognition is increasing continuously due to its reliability, accuracy and uniqueness. To improve the overall recognition rate and performance of iris recognition system, the researchers have to work in different aspects like unconstrained environment, noisy images as well as blurred images. This paper overviews the different steps involve into iris recognition system as well as various methodology presents in the iris recognition system.
\end{abstract}

\section{Keywords}

Acquisition, Localization, Iris recognition, Feature Extraction, Matching.

\section{INTRODUCTION}

The iris is a thin, circular structure in the eye, accountable for controlling the diameter and the quantity of light reaching the retina by pupil size. The color of the iris provides the eye its color. According to optical terms, the pupil is the eye's opening and the iris is the diaphragm that attends as the aperture stop. Iris Recognition has attained a great attention in past few years due to its unique features such as ridges, freckles, rings, furrows and complex pattern and thus it poses a great degree of uncertainty [1]. All the individuals have unique eye pattern [2]. The Iris Recognition is one of the most promising biometric identification technologies with high individuality and steadiness, noninvasiveness, antifalsification and many other advanced qualities [3].

The concept of iris recognition was proposed as a reliable Biometric for the first time in 1987.Many powerful iris recognition system algorithms are proposed by many researchers like John Daugman, K.W. Bowyer, and Wildes etc. Iris recognition system collects iris images using Near Infrared imaging (NIR). The superiority of the NIR-based iris recognition technologies has been practically engaged in very large scale applications, such as in Aadhar project [8] to identify millions of citizens in India, or in border-crossing control system in UAE [4]. The iris recognition describes of main four steps after doing image acquisition. They are segmentation, normalization, feature extraction and feature equivalent [3]. The segmentation process of iris recognition is to separate the actual iris region in a digital eye image. The iris region can be approached by two circles, one for outer iris/sclera boundary and the other for inner iris/pupil border. The eyelids, eyelashes and specular reflections can occur within the iris region that corrupts the iris pattern. So techniques are required to isolate and exclude these corruptions as well as locating the circular iris region.
The normalization stage is to transform the iris region into fixed dimensions in order to allow comparisons. This is for reducing the dimensional inconsistencies. After normalization we will get iris regions of the same constant dimensions. Two photographs of the same iris under different conditions will have characteristic features at the same spatial location. The most discriminating information present in an individual's iris pattern must be extracted and compared, for the accurate and robust recognition of individuals. Only the significant features of the iris must be encoded in feature extraction, so that comparisons between templates can be made. Iris images to create a biometric template. The iris is categorized into such major regions: first is the pupillary zone is the inner region whose edge forms the border of the pupil. Second is the ciliary zone is the rest of the iris that spreads to its origin at the ciliary body.

This paper gives detail overview of Iris recognition system using geometrical key based encoding techniques. It also insides the different performance parameter for recognition rate.

\section{LITERATURE SURVEY}

Recently numbers of techniques are used for iris recognition techniques in various applications. Each technique produces different artifacts and results. Ramli et al. [5] projected algorithm based on $\mathrm{Hu}$ invariant instant. CASIA dataset used by the authors which consists of 108 courses, but 1 mage per iris and 100 images were used in the training. Azizi and Reza [4] have used phase information for iris recognition. In the segmentation step, linear Hough transform method is used, two lines are drawn, first a line to the upper eyelid and lower eyelid then a second line is drawn which intersect the pupil boundary. Eyelashes are separated with the help of ID Gabor filter and isolate iris complex pattern area. Using Log Gabor filter for segmentation used by Lagree and Bowyer [6]. Segmented images to obtain the desired portion and disguise the area containing eyelid portion of iris. Various filters are used to build a Feature Vector like "Spot detector" and "Line detector". For all iris images this vector is calculated.

According to the Rose theory [7] iris recognition divides into five category i.e image gaining, segmentation, normalization, matching and encoding. Look into the acquisition series of iris images are captured and chosen only quality images have maximum information. Another detection method known as integro-differential" operator iris recognition is the most critical section because accurate detection increases accuracy of the system. During Normalization method iris of same area is obtained for similar with additional irises. In this paper need to more enhance iris image with the help of encoding. It starts after getting enhanced image first need to extract iris textural features using "2D Gabor Filter" then encoded each texture as "iris code" 2D binary code.at the last performing matching by comparing 2 iris codes named as hamming Distance. 


\section{IMAGE PREPROCESSING}

Iris recognition system is below fig.1.The first step of the system is to capture the eye image, captured image is then send for preprocessing. The main aim of preprocessing is to check the quality of captured images, then quality of the images if it is good then first locate the iris in captured image and if the quality of images is not good then need to do improvement. Iris image pre processing is categorized into three steps: iris localization, iris normalization and image improvement.

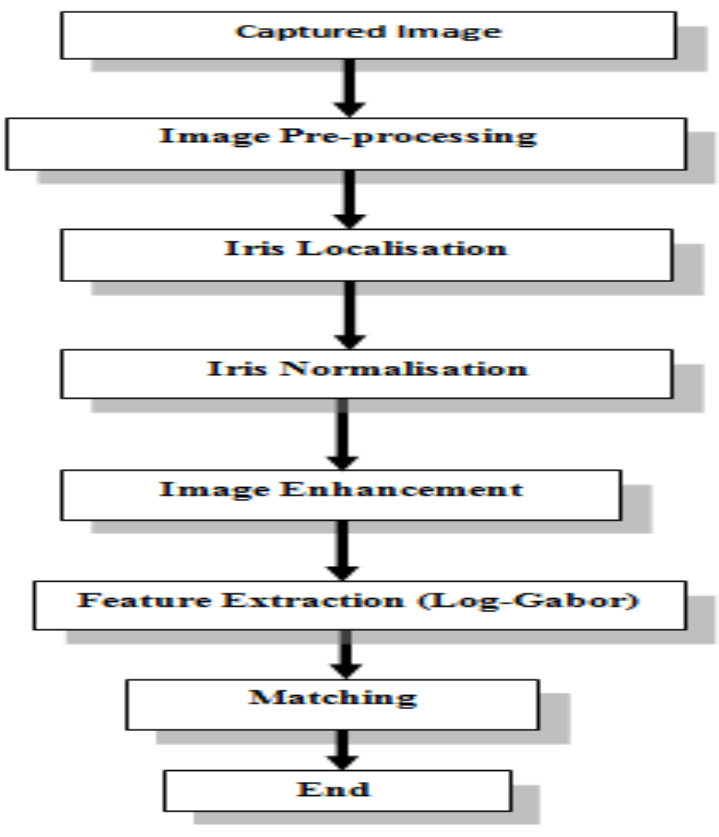

Figure 1: steps of Iris recognition system

Center of the pupil is poor quality to iris. Pupil radius ranges from 0.1 to 0.8 of iris radius [1].Isolation of the region from the eye image is mainly concerned with acquisition of image. Aim of image acquisition is to get quality image.

\subsection{Iris Localization}

Localization detects the inner and outer boundaries of the iris. The most important step is both the inner and outer iris boundaries can be approximately modeled as circles and correct iris portion is necessary to generate templates [8]. Iris localization is classified into four different techniques discussed in this section.

\subsubsection{Traditional Methods}

This method of iris localization use to locate iris boundaries using various edge detection operators. However in the iris boundary they also contain some parts of eyelashes which results in the faulty iris segmentation. All methods were based on various assumptions that iris and pupil are always circular and thus one can segment the region accordingly which is nothing but to detect outer and inner boundaries [9].

The customary methods used for iris localization are given below:

\subsubsection{Canny Edge Detector:}

Canny edge recognition is used to detect the edges of iris images. The edges are detected as they are good and possible marks of the real edges in the images [10]. During canny edge detection, first, noise is abolished from captured image. Then edge strength is found and edge direction is defined.
Double thresholding is applied using hysteresis method to improve edge detection accuracy [10].

\subsubsection{Integro differential operator}

Daugman's integro-differential operator is used to first find the coordinates of the iris boundary [8]. This operator finds the (J) maximum pixel intensity value change by searching the image within the defined radius by using a circular integral centered on the point $(\mathrm{x} 0, \mathrm{y} 0)$, with radius $\mathrm{r}$ of the radial derivate of the original image blurred with a gaussian kernel G[8].Equation (1) of integro differential operator given below:

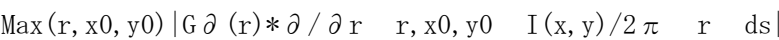
(1)

\subsubsection{Circular Hough Transform}

The general Hough convert can be used on any kind of shape although the complexity of the transformation increases with the number of parameters needed for describing the shape of an image. Circular Hough convert is used to detect the iris boundaries. First, the conversion of point in each (x, y) space is done into the corresponding Hough parameter space [12]. The circle is meeker to represent in parameter space, matched to the line, since the parameter of the circle can be straight transferred to the parameter space points $(\mathrm{a}, \mathrm{b}, \mathrm{r})$. As it can be seen the circle to get three parameter $r$, $a$ and $b$, where $a \& b$ are the center of the circle in the direction $\mathrm{x} \& \mathrm{y}$ respectively and $\mathrm{r}$ is the radius [12].

Hough transform votes are cast in three direction accumulator. To select the proper scale of edge analysis and to smooth the image Gaussian filter is applied [8]. More the number of cells more will be the accuracy, Accuracy depends upon the number of accumulator cells.

\subsubsection{Bisection method}

In this Method, the center of the pupil is located by using bisection method. An iris image is to extract the edge information by applying edge detection. The perpendicular lines are drawn through the center point y by applying bisection method [13][14]. The radius is increased within a certain range and virtual circle is drawn with reference to the center of the pupil. It is affected by the non-uniform illuminations and glasses reflections.

\subsection{Iris Normalization}

The process of normalization is remapping images from Cartesian coordinates to polar coordinates and create fixed dimension iris images [16].In iris image Pupil size may vary in illumination due to the contraction of pupil in light. A person's same eye the different iris images of may appear different due to conditions under which they were taken. By using fixed parameter interval Daugman normalized the iris image by representing the image in a doubly dimensionless pseudo polar coordinate system [1][17]. The segmented iris region is normalized in order to obtain a fixed number of features from the iris regardless of its spatial resolution. And Map the iris to a dimensionless fixed coordinated system that is invariant to size changes, i.e. pupil dilation and iris size changes.

\subsubsection{Homogenous rubber sheet model}

The Rubber Sheet model maps the coordinates of each Cartesian point from the segmented iris region to polar coordinates $(r, \theta)$ where $r$ ranges from 0 to 1 and $\theta$ ranges from $02 \pi$.In different eye images from the same subject, changes in the iris pattern occur due to pupil dilation or constriction. The 
alteration to a dimensionless form also ensures the iris pattern is not prejudiced by magnification and image distance acquisition factors. John Daugman's rubber sheet model ensures the proper handling of the matter due to the particulars of the iris. It accounts size inconsistencies and pupil dilation, but does not give back for rotating inconsistencies.

\subsection{Image Enhancement}

The process of improving the advantage and contrast of an image is known as image enhancement. The normalized image needs to advance because usually it is of low contrast non-uniform illumination caused by the light source position. It does not affect for matching stages and further dealing out. The whole image of intensity variation is approximated to get well distributed texture figure [18].Further dealing out is accomplished by the histogram equalization. The contrasts of the image improve this processing which is then used for outline matching and feature extraction [18].

\subsection{Feature Extraction}

Feature extraction is the first and most important step in image classification. Latest available image classification methods apply hand-crafted features, which are not adaptive for a variety of image domain. Feature extraction is the main part of iris recognition system.

\subsubsection{Log gabor Filter}

Iris features extracted by using 2D Gabor filters are used in both [1] and [2]. 1D Log-Gabor filters are convolved the image because the first reason is they provide phase information. And second is they are constructed by modulating sine's and cosines waves with a Gaussian filter which makes them useful for localizing in space and frequency. Phase information of each Iris pattern is extracted with the help of quadrature 2D Gabor wavelets. The phase information is quantized into four quadrants in the complex plane.

\subsubsection{Waelet Transform}

The wavelet transform decomposes the iris region into components with different resolutions [1]. Feature are localized in both space and frequency domains with varying window sizes. It has both space resolution and frequency resolution. Advantage of wavelet transform over Fourier transform is that the Wavelet filters have a bank and it applied to the iris normalized region [1].

\subsubsection{Laplacian of Gaussian filter}

Laplacian of Gaussian filters are used to encode feature by decomposing the iris region. Filtered image is realized as a laplacian pyramid. The Laplacian pyramid is constructed with four levels to generate a compact biometric template. Approach compresses the data to obtain important data. It can be kept and processed effectively.

\subsubsection{Discrete Cosine Transform}

Iris is coded in coefficients of rectangular patches ba225j/sed on differences of Discrete Cosine Transform (DCT).To reduce the effect of noise, the average over width is windowed using a Hanning window[8]. DCT and a similar Hanning window are applied to the patch alongside its length [8]. The differences of adjacent patches between the DCT coefficients are obtained. From the zero crossings of the differences between the DCT coefficients, a binary crossing template is generated. This coding method has low complexity and good
Inter-class separation. It is superior to other approaches in speed and accuracy both.

\subsubsection{Geometric key Based Iris Encoding}

This paper proposes the concept of geometric key, which is the set of coordinate-pairs It is randomly (pseudorandom) generated and exclusively assigned to each subject enrolled into the system [20]. Such geometric key can be measured as unique (personalized) to each of the subjects and it uniquely defines the way how the iris features are encoded from the restricted regions. Such adapted strategy is expected to get better the separability between the inter-class and intra-class distributions and so the improvement in iris recognition act can be expected [20]. Better accommodating for the imaging variations is strength of localized iris encoding and the global iris encoding strategy has its strength in effective encoding of less noisy iris region pixels.

\subsection{Template Matching}

The templates generated from the feature withdrawal stage need analogous corresponding metric. The matching metric compares the similarity between the templates. A threshold is put to make a distinction between intraclass and interclass comparisons.

\subsubsection{Hamming Distance}

Hamming distance is defined as the small measure of deviation among two binary templates [1], [17]. A zero value would symbolize a perfect competition. The Hamming Distance (HD) is the sum of differing bits of two iris templates divided by the total number of bits. HD $=0$ if two patterns are from the same Iris. Since errors can occur throughout segmentation and normalization, HD should be close to 0 for the similar irises but rarely be equal to 0 . To account for rotational inconsistencies, when the HD of two templates is considered, one template is shifted left and right bit wise from the calculated HD values, only the lowest is taken, since this corresponds to the best match between two templates.

\subsubsection{Weighted Euclidean distance}

It is used to compare two templates to identify an iris [19]. The templates are self-possessed of integer values. Weighted Euclidean Distance is a measure of comparison between two templates. By using Pythagorean Theorem to obtain the distance between two points it is considered. An iris template is compared with all templates in the database. Matching two templates are for minimum Weighted Euclidean Distance.

\section{PERFORMANCE PARMETER}

The performance parameter could be recognition rate, time complexity and space complexity. Recognition performance has many measurement standards. The most important and popular formula are recognition rate in equation (2).

Recognition Rate $=$ Number of recognized images

$$
\text { Number of testing images }
$$

Recognition Rate or accuracy can be defined as the percentage of total successfully classified samples out of total tested samples. Accuracy can also be measured in terms of FAR (False Acceptance Rate) and FRR (False Rejection Rate) False Acceptance Rate is the percentage of total other person samples those were misclassified as the current samples. False Rejection Rate is the percentage of two samples those were recognized as another person than the true person. 


\section{SUMMARY OF IRIS RECOGNITION}

\section{TECHNIUES}

\begin{tabular}{|c|c|c|c|c|}
\hline No & Recognition Methods & Specified area & Advantages & Disadvantages \\
\hline 1 & 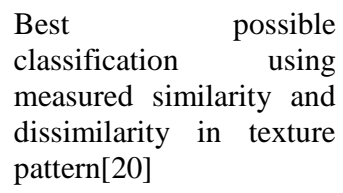 & $\begin{array}{l}\text { Iris texture pattern identification was } \\
\text { human defined }\end{array}$ & Strongly classification & $\begin{array}{l}\text { It is very active for small } \\
\text { dataset } \\
\text { Accuracy: } 80 \%\end{array}$ \\
\hline 2 & $\begin{array}{l}\text { Build feature vector } \\
\text { applying for "spot" and } \\
\text { "Line" detector filters [1] }\end{array}$ & $\begin{array}{l}\text { Civilization prediction based on iris } \\
\text { texture }\end{array}$ & $\begin{array}{l}\text { Accuracy is higher through } \\
\text { SMO by using WEKA }\end{array}$ & $\begin{array}{l}\text { Local features has not been } \\
\text { evaluated } \\
\text { Accuracy: } 75 \%\end{array}$ \\
\hline 3 & $\begin{array}{l}\text { Feature Extraction by } \\
\text { using through transform } \\
\text { and feature selection } \\
\text { through PCA and ICA } \\
\text { algorithm [17] }\end{array}$ & $\begin{array}{l}\text { Application of phase base approach } \\
\text { for iris feature extraction }\end{array}$ & $\begin{array}{l}\text { Template matching time } \\
\text { decreases and classification } \\
\text { accuracy increases }\end{array}$ & $\begin{array}{l}\text { Noise damage calculation } \\
\text { procedure } \\
\text { Accuracy: } 80 \%\end{array}$ \\
\hline 4 & $\begin{array}{l}\text { Grouping between left } \\
\text { and right eye [3] }\end{array}$ & Iris images are with Noise & $\begin{array}{l}\text { Distinguish between left and } \\
\text { right eye, performance and } \\
\text { accuracy increases }\end{array}$ & $\begin{array}{l}\text { Input eye cannot be } \\
\text { distinguish as left and right } \\
\text { Accuracy: } 70 \%\end{array}$ \\
\hline 5 & $\begin{array}{l}\text { Removal of eyelid and } \\
\text { eyelashes [17] }\end{array}$ & High speed iris segmentation & $\begin{array}{l}\text { It is very effective for noisy } \\
\text { images }\end{array}$ & $\begin{array}{l}\text { The accuracy of the system is } \\
\text { affecting as more reflection } n \\
\text { pupil and iris region } \\
\text { Accuracy: } 85 \%\end{array}$ \\
\hline 6 & $\begin{array}{l}\text { Authentication on } \\
\text { algorithm based on } \\
\text { segmentation process[5] }\end{array}$ & $\begin{array}{l}\text { Automated recognition and } \\
\text { performance measurement }\end{array}$ & $\begin{array}{l}\text { Well-organized matching } \\
\text { accuracy }\end{array}$ & $\begin{array}{l}\text { It is applicable for small } \\
\text { dataset } \\
\text { Accuracy: } 60 \%\end{array}$ \\
\hline 7 & $\begin{array}{l}\text { It was combining for } \\
\text { multiple techniques }\end{array}$ & Method for iris localization & $\begin{array}{l}\text { Performance and accuracy } \\
\text { increases }\end{array}$ & $\begin{array}{l}\text { Each iris image has require } \\
\text { manual editing of pupil area } \\
\text { Accuracy:(method not clear) }\end{array}$ \\
\hline 8 & $\begin{array}{lr}\text { Iris } & \text { identification } \\
\text { challenges } & \text { and } \\
\text { multispectral recognition }\end{array}$ & Iris recognition & $\begin{array}{l}\text { For Iris recognition problem } \\
\text { area identification }\end{array}$ & $\begin{array}{l}\text { In this at primary level discuss } \\
\text { many methods } \\
\text { Accuracy:(method not clear) }\end{array}$ \\
\hline 9 & $\begin{array}{l}\text { Iris is divided into six } \\
\text { regions using } \\
\text { thresholding [1] }\end{array}$ & Non-cooperative iris recognition & $\begin{array}{l}\text { After comparison iris regions } \\
\text { false rejection ratio has been } \\
\text { decreased }\end{array}$ & $\begin{array}{l}\text { To extracting small textural } \\
\text { information did high } \\
\text { calculation } \\
\text { Accuracy: } 65 \%\end{array}$ \\
\hline 10 & $\begin{array}{l}\text { Difference between } \\
\text { global and local iris } \\
\text { features[3] }\end{array}$ & $\begin{array}{l}\text { Iris recognition system has new } \\
\text { approach }\end{array}$ & $\begin{array}{l}\text { Not dependent on } \\
\text { normalization }\end{array}$ & $\begin{array}{l}\text { Not able to follow the } \\
\text { traditional } \\
\text { method } \\
\text { Accognition }\end{array}$ \\
\hline 11 & $\begin{array}{l}\text { Getting iris texture } \\
\text { feature by using nine } \\
\text { filters[17] }\end{array}$ & $\begin{array}{l}\text { From iris texture need to predict } \\
\text { age group }\end{array}$ & Aging factor informed & $\begin{array}{l}\text { Accuracy become less } \\
\text { Accuracy: } 60 \%\end{array}$ \\
\hline
\end{tabular}




\section{CONCLUSION}

The various Iris recognition systems are analyzed for selecting appropriate techniques in each step to develop a more accurate biometric System. For Localization edge detection and Hough transform methods are used. Further Daugman's Rubber Sheet Method is used for Normalization. Log Gabor feature is used for Iris Encoding. The recognition rates of various techniques are compared. It can be increased with more no. of training data sets and appropriate algorithm.

\section{ACKNOWLEDGMENTS}

Authors would like to thank to the institute authorities for their kind support without which this work could not have been conducted.

\section{REFERENCES}

[1] John Daugman, "Statistical richness of visual phase information: updateon recognizing persons by iris patterns," International Journal of Computer Vision, vol. 45, no.1, pp.25-38, 2001.

[2] John Daugmann, "How iris recognition works," IEEE Trans. Circuits Syst. Video Technol. ,vol.14, no.1, pp. $21-30,2004$

[3] Shaabad A.Sahmoud and Ibrahim S. Abuhaiba, "Efficient iris segmentation method in unconstrained environments," Pattern Recognition, vol.46, no.12, pp. 3174-3185, 2013.

[4] Mohamad-Ramli, N.A., M.S. Kamarudin and A. Joret, 2008. "Iris Recognition for Personal Identification", The International Conference on Electrical Engineering (ICEE), July 6-10, 2008, OKINAWA, JAPAN.

[5] AziziA. and H. Reza, 2009. "Efficient IRIS Recognition through Improvement of Feature Extraction and subset Selection", (IJCSIS) International Journal of Computer Science and Information Security, 2: 1.

[6] Lagree, S. and K.W. Bowyer, 2010. "Ethnicity prediction based on iris texture features", In: 22nd Midwest Artificial Intelligence and Cognitive Science Conference (MAICS).

[7] Richard Yew Fatt Ng, Yong Haur Tay and Kai Ming Mok, "A review of iris recognition algorithms," IEEE Trans. Information Technol., vol.2, pp. 1-7, 2008.

[8] Jing Huang, Xinge You, Yuan Yan Tang, Liang Du and Yaun Yaun, "A novel iris segmentation using radial- suppression edge detection," Signal Processing, vol.89, no.12, pp.2630-2643, 2009.

[9] J.Canny, "A computational approach to edge detection," IEEE Trans. Pattern Analysis and Machine Intelligence, vol.8, no.6, pp.679-698, 1986

[10] Annapoorani, G., R. Krishnamoorthi, P.G. Jeya and Petchiammal, 2010. "Accurate and Fast Iris Segmentation", International Journal of Engineering Science and Technology, 2(6): 1492-1499.

[11] Naphtali Rishe and Jean Andrian, "A highly accurate and computationally efficient approach for unconstrained iris segmentation," Image and Vision Computing, vol. 28, no.2, pp. 261-269, 2010

[12] S. Lim, K. Lee, O. Byeon, and T.Kim (2001). "Efficient Iris Recognition through Improvement of Feature Vector and Classifier", ETRI Journal, vol. 23, no.2, pp. 61-70.

[13] H. Sung, J. Lim, J. Park, and Y. Lee (2004). "Iris Recognition Using Collarette Boundary Localization", Proceedings of the 17th International Conference on Pattern Recognition, vol. 4, pp. 857-860.

[14] M. S. Crouse, R. D. Nowak, and R. G. Baraniuk, 1998 "Wavelet-based statistical signal processing using hidden Markov models", IEEE Trans. Signal Process., vol. 46, no. 4, pp. 886-902.

[15] D.M. Rankin, B.W. Scotney, P.J. Morrow and B.K. Pierscionek, "Iris recognition failure over time: The effects of texture," Pattern Recognition, vol.45, no.1, pp.145-150, 2012.

[16] John Daugman "High confidence visual recognition of persons by a test of statistical independence," IEEE Trans. Pattern Analysis and Machine Intelligence, vol.15, no.11, 1993.

[17] L. Ma, T. Tan, Y. Wang and D. Zhang, "Personal identification based on iris texture analysis," IEEE Trans. Pattern Analysis and Machine Intelligence, vol.25, no.12, pp.1519-1533, 2004.

[18] Y. Zhu, T. Tan, and Y. Wang (2000). "Biometric Personal Identification Based on Iris Patterns", Proceedings of the 15th International Conference on Pattern Recognition, vol. 2, pp. 2801-2804. 